\title{
Biologia reprodutiva de Heros efasciatus Heckel, 1840 (Pisces, Cichlidae) na Reserva de Desenvolvimento Sustentável Amanã-AM, visando seu manejo sustentável
}

\author{
Jana Menegassi del FAVERO ${ }^{1}$, Paulo dos Santos POMPEU, Ana Carolina PRADO-VALLADARES 3
}

\begin{abstract}
RESUMO
Com o principal objetivo de fornecer ferramentas para auxiliar na implementação do manejo sustentável de peixes ornamentais na Reserva de Desenvolvimento Sustentável Amaná, Amazonas, foi realizado o estudo da biologia reprodutiva de Heros efasciatus Heckel, 1840, um ciclídeo com potencial ornamental e com poucos trabalhos sobre a sua biologia e ecologia, apesar de já ser comercializado em algumas regióes amazônicas. Coletas bimestrais foram realizadas de fevereiro de 2006 a janeiro de 2007 em dez igarapés contribuintes do Lago Amanã e Urini, sendo utilizados três aparelhos de pesca (rede de arrasto, rapiché e armadilha tipo matapi) e ainda galhadas artificiais nas amostragens realizadas próximas aos lagos. Foram capturados 140 exemplares de $H$. efasciatus, sendo 50 fêmeas, 42 machos, e 46 indivíduos cujo sexo não foi identificado devido ao pequeno tamanho. O tipo de crescimento encontrado foi isométrico, sendo que o maior indivíduo observado apresentava $174 \mathrm{~mm}$ e o menor $14 \mathrm{~mm}$. Os resultados encontrados auxiliarão na adoção de medidas de manejo, como a determinação de tamanhos mínimos de captura, superiores aos tamanhos médios de maturação ( $97 \mathrm{~mm}$ para as fêmeas) e o estabelecimento de períodos de defeso durante a época de sua reprodução (outubro a janeiro). A pequena abundância de indivíduos da espécie, quando comparada com o total de exemplares capturados (apenas 0,07\%) e a baixa fecundidade média, de 2502 ovócitos, indica que se deve trabalhar anualmente apenas com um pequeno número de indivíduos, a fim de garantir a continuidade do estoque.
\end{abstract}

PALAVRAS-CHAVE: Amazonas, Cichlidae, peixe ornamental, reprodução de peixes.

\section{Reproductive biology of Heros efasciatus Heckel,1840 (Pisces, Cichlidae) in the Amanã Sustainable Reserve (Amazonas, Brazil), aiming at sustainable management of the species}

\begin{abstract}
The aim if the present work is to supply basic information that may help the management of ornamental fishes in the Amanã Sustainable Reserve, State of Amazonas, Brazi. A study of the reproductive biology of Heros efasciatus, a cichlid fish with ornamental potential, was performed using specimens collected bi-monthly between February 2006 and January 2007 in ten creeks contributing to Lakes Amanã and Urini. The sample collection gear included hand net, seine and traps. In addition, artificial tree branches were used for sampling near the lakes. A total of 140 specimens of $H$. efasciatus were captured, which corresponded to only $0.07 \%$ of all specimens collected. Of this total, 50 were male, 42 were female, and 46 individuals could not be sexed due to their small size. The type of growth founded was isometric; with individuals' sizes varying from $14 \mathrm{~mm}$ to $174 \mathrm{~mm}$. Average fecundity was estimated at 2,502 oocytes. The results of this study will help in implementing several sustainable management measures, such as establishing a minimum size for capture (higher than $97 \mathrm{~mm}$ ) and imposing a no-fishing restriction during the spawning season of this species (from October till January). To ensure stock continuity due to the low density and low fecundity of $H$. efasciatus, we suggest that exploitation of this species for ornamental trade be kept to a small number of individuals per year.
\end{abstract}

KEYWORDS: Amazonas, Cichlidae, Ornamental fish, reproductive biology.

1 Instituto Oceanográfico, Universidade de São Paulo. E-mail: janamdf@yahoo.com.br

2 Universidade Federal de Lavras. E-mail: pompeu@ufla.br

${ }^{3}$ Instituto de Desenvolvimento Sustentável Mamirauá. E-mail: carolvalladares@mamiraua.org.br 


\section{INTRODUÇÃO}

Os ciclídeos são os mais populares peixes de aquário, pois possuem alta diversidade de coloração, forma, tamanho e comportamento (Goldstein 1973), sendo a bacia amazônica o maior fornecedor mundial (Lowe-McConnell 1999). São adaptados a uma diversidade de tipos de reproduçáo, variando de desova em substrato com pequeno cuidado parental até desovas com vigorosa proteçấo, como aquelas em que a mãe, ou o pai, ou ambos incubam os ovos na boca além do nascimento e reabsorção do vitelo, até que a cria seja capaz de se defender sozinha. Tipicamente, um ou ambos os pais se encarregam de algum grau de proteção indiferente do tamanho da prole. A maioria das espécies de ciclídeos possui desova parcelada, chegando a desovar até quatro vezes durante o ciclo reprodutivo (Keenleyside 1991). O mecanismo de desenvolvimento ovocitário é do tipo "sincrônico em mais de dois grupos", em que os lotes maduros dos ovócitos são eliminados sucessivamente dentro de um dado período reprodutivo (Vazzoler 1996).

Dentre os ciclídeos amazônicos, Heros efasciatus Heckel, 1840 é bentopelágico e vive em ambientes de $\mathrm{pH}$ básico $(\mathrm{pH}$ 7) com temperatura variando de 24 a $32^{\circ} \mathrm{C}$. Sua distribuição é restrita à bacia do rio Amazonas, sendo encontrado em tributários do Ucayali e rio Amazonas no Peru e do SolimóesAmazonas no Brasil. Tem sido capturado esporadicamente em ambientes lênticos como as zonas alagáveis e estuários, ao longo dos bancos de vegetação ribeirinha. É onívora, mas predominantemente frugívora, principalmente no período de alagamento da floresta (Froese e Pauly 2009). É um peixe pouco comercializado, porém alguns trabalhos relatam sua importância como espécie ornamental (Cáuper 2006). Heros efasciatus também é citado na lista de espécies de peixes encontradas no mercado de Manaus-AM para o consumo de carne (Batista e Petrere Júnior 2003). Possui fecundação externa, com grande cuidado parental. Mais de 1000 ovos são depositados em rochas lisas ou em raízes. Ambos os pais participam do cuidado dos ovos e das larvas (Froese e Pauly 2009).

O Projeto "Peixes Ornamentais", do Instituto de Desenvolvimento Sustentável Mamirauá, foi criado com o objetivo de proteger a diversidade de peixes ornamentais da Amazônia, desenvolvendo um projeto piloto na Reserva de Desenvolvimento Sustentável Mamirauá (RDSM) e na Reserva de Desenvolvimento Sustentável Amanã (RDSA), tentando estabelecer um comércio sustentável de peixes ornamentais de água doce. A possível realização de tal comércio resultará em benefícios econômicos diretos às comunidades e, junto com o estabelecimento de um sistema sustentável, assegurará a proteção em longo prazo da diversidade dos peixes dentro das reservas. Para atingir tal objetivo, faz-se necessário, além de se conhecer a composição da ictiofauna das reservas, a compreensão do ciclo de vida e da biologia das espécies que provavelmente serão manejadas. Fazendo parte deste projeto, o presente trabalho teve como objetivo caracterizar a biologia reprodutiva do acará-roxo, $H$. efasciatus, buscando informaçôes biológicas que contribuirão para a futura elaboraçáo de um plano de manejo na Reserva de Desenvolvimento Sustentável Amaná.

\section{MATERIAIS E MÉTODOS}

\section{ÁREA DE ESTUDO}

A Reserva de Desenvolvimento Sustentável Amanã é uma das maiores áreas protegidas em floresta tropical na América do Sul, com cerca de 2.350.000 hectares. Está localizada na bacia central do Amazonas entre os rios Negro e Japurá (Figura 1) (Nogueira 1997).

A pluviosidade média anual na regiáo é de aproximadamente $2.850 \mathrm{~mm}$ e mostra uma distinta sazonalidade. A estação mais chuvosa, geralmente vai de dezembro até março e alcança até três vezes os níveis de precipitaçáo da estação menos chuvosa (denominada na regiâo de estaçáo seca) que vai de julho a outubro (Fleck 2004). A temperatura diária média é $29,5^{\circ} \mathrm{C}$, sendo essa média praticamente constante em todos os meses, variando apenas $1,8^{\circ} \mathrm{C}$ (Ayres 1993).

O lago Amanã é o maior lago da RDSA, atingindo aproximadamente $40 \mathrm{~km}$ de comprimento e possuindo uma área de mais de $100 \mathrm{~km}^{2}$. É um lago de água preta, pobre em nutrientes. Porém ele recebe água branca, rica em nutrientes, na sua seção mais baixa durante a cheia. $\mathrm{O}$ pulso de enchente é marcável, resultando em extensas áreas de florestas alagadas sazonalmente (Ayres 1993).

\section{MÉTODOS}

As coletas realizadas faziam parte de um projeto de levantamento da ictiofauna da regiāo, de onde foram retirados os indivíduos para análise. Foram amostrados cinco igarapés situados em ambientes de terra firme e cinco próximos às margens do lago Amanã e Urini (Figura 1), sendo realizadas seis coletas bimestrais ao longo de um ano (fevereiro de 2006 a janeiro de 2007). Nas amostragens nos igarapés de terra firme, foram utilizados três aparelhos de pesca: redinha (arrasto), rapiché (puçá) e armadilha tipo matapi (covo). A rede de arrasto media dez metros de comprimento por três de altura. Com este aparelho realizaram-se dois cercos em cada um dos pontos de coleta. Os rapichés possuíam a forma quadrada medindo $50 \times 50 \mathrm{~cm}$. Com este aparelho fez-se 200 lances seguidos nas proximidades dos pontos de coleta. Foram usadas também cinco armadilhas tipo matapi por ponto de coleta, que permaneceram 24 horas na água e foram retiradas apenas uma vez. As aberturas da boca destas armadilhas começavam com $25 \mathrm{~cm}$ de diâmetro e afunilavam até atingirem cinco 


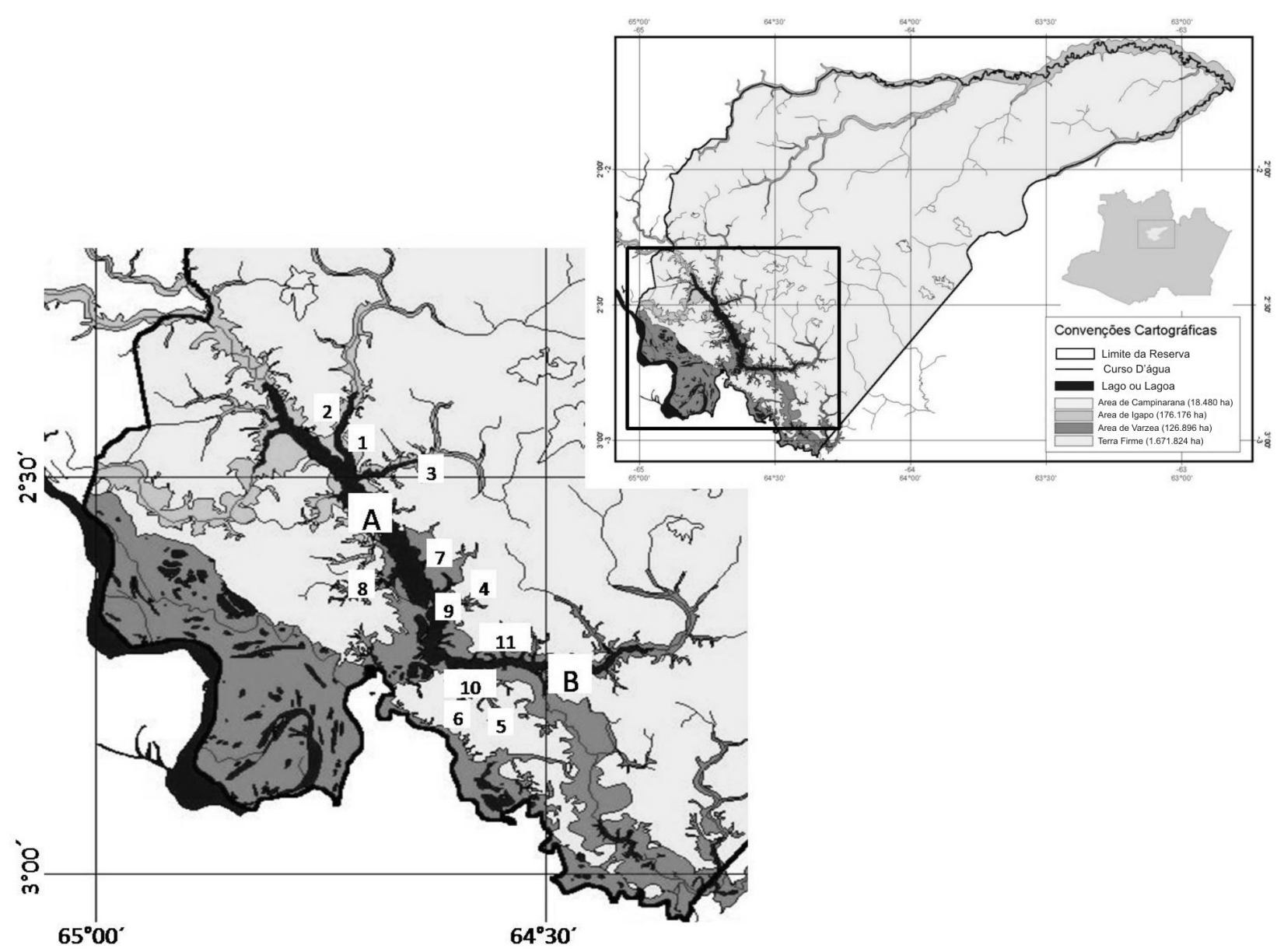

Figura 1 - Mapa da Reserva de Desenvolvimento Sustentável Amanã no estado do Amazonas ressaltando os locais de coleta. As letras correspondem ao Lago Amanã (A) e Urini (B) e os números aos igarapés amostrados ( $1=$ Veado, $2=$ Queimada, $3=$ Ubim, $4=$ Cacau, $5=$ Guariba, $6=$ Tirirical, $7=$ Samaúma, $8=$ Açu, $9=$ Cacau, $10=$ Araueri, $11=$ Branco). Nos igarapés de 7 a 11 foram utilizadas "galhadas" nas amostragens.

$\mathrm{cm}$; a distância entre as duas bocas era de $80 \mathrm{~cm}$. No interior das mesmas foram utilizadas carne em conserva para atrair os peixes. Além disso, nas coletas realizadas próximas ao lago Amanã e Urini, foram utilizadas galhadas artificiais, que foram amostradas depois de três dias com uma rede de arrasto.

Todos os indivíduos coletados foram fixados em formol 4\% e levados para o laboratório do Instituto Mamirauá. Cada exemplar de Heros efasciatus, após serem identificados, pesados e medidos (comprimento padrão), foram dissecados para identificação macroscópica do sexo, pesagem das gônadas e do estádio de maturação segundo Vazzoler (1996), que classificou as gônadas em cinco estádios: imaturo, em maturação, maduro, esvaziado e em repouso. Como o estádio "repouso" é de difícil classificação sem microscopia, pois a gônada nesta situação se assemelha com a imatura, foi calculado o tamanho médio em que os indivíduos iniciavam a maturação e todos aqueles julgados imaturos acima de tal tamanho foram considerados em repouso (estádio entre o fim de uma desova e o início de uma nova maturaçấo gonadal). O comprimento médio de primeira maturaçáo $\left(\mathrm{L}_{50}\right)$ foi estimado seguindo Vazzoler (1996) e corresponde àquele com o qual 50\% dos indivíduos iniciam seu ciclo reprodutivo.

$\mathrm{Na}$ distinção sexual, muitas vezes contou-se com auxílio de um estereomicroscópio, principalmente nas gônadas imaturas, devido à dificuldade de se visualizar pequenos ovócitos a olho desarmado.

Para os cálculos de fecundidade e tipo de desova foram selecionadas as sete gônadas de fêmeas maduras encontradas, coletas no mês de outubro, as quais foram abertas em placa de Petri e, com o auxílio de um estereomicroscópio, seus ovócitos tiveram os diâmetros medidos (paquímetro digital marca Digimes, modelo 100.176BL) sendo então separados em lotes de três tamanhos (T1: 0,10-0,50mm; T2: 0,501,00mm; T3: 1,00-1,50mm), contando assim o número de ovócitos em cada lote. 
A relação peso-comprimento foi calculada através da equação $\mathrm{W}=\mathrm{aL}^{\mathrm{b}}$ (Le Cren 1951), transformada logaritmicamente em: $\log \mathrm{W}=\log \mathrm{a}+\mathrm{b} \log \mathrm{L}$, onde $\mathrm{W}$ corresponde ao peso do peixe em gramas e $\mathrm{L}$ ao seu comprimento em milímetros. Com o objetivo de verificar se $\mathrm{a}$ constante $\mathrm{b}$ foi significativamente diferente do valor de crescimento isométrico $(b=3)$, foi aplicado o teste $t$ de Student $(\mathrm{H} 0: \mathrm{b}=3)$ com nível de confiança de p de 5\% $(a=0.05)$ (Sokal e Rohlf 1987). Diferenças na proporção sexual foram testadas através do teste $G$ (Zar 1999).

Foi ainda calculado o fator de condição (k) para crescimento alométrico expresso por: $\mathrm{k}=\mathrm{Wt} / \mathrm{Lt}^{\mathrm{b}}$, onde $\mathrm{Wt}=$ peso total do indivíduo, $\mathrm{Lt}=$ comprimento do indivíduo $\mathrm{e}$ $\mathrm{b}=$ coeficiente de regressão entre $\mathrm{Wt} / \mathrm{Lt}$, bem como o Índice Gonadal (IG) através da expressão: $\mathrm{Wg} / \mathrm{Lt} t^{\mathrm{b}}$, onde $\mathrm{Wg}=$ peso da gônada e a relação gonadossomática (RGS), que expressa a porcentagem que as gônadas representam do peso total (Vazzoller 1996). Devido ao pequeno número de fêmeas coletadas, todos os calculos citados acima foram feitos para os sexos agrupados. Diferenças no fator de condição $(\mathrm{k})$ entre os meses de coleta foram testadas através do teste de Kruskal-Wallis.

\section{RESULTADOS}

Ao longo do período de coletas foram capturados 140 exemplares de $H$. efasciatus, que representaram $0,07 \%$ do total de peixes amostrados (Tabela 1).

Tabela 1- Quantidade em número de Heros efasciatus, do total de peixes capturados e a abundância relativa de $H$. efasciatus por período de coleta.

\begin{tabular}{lccc}
\hline Mês/ano & H. efasciatus capturados & Total Capturado & Proporção (\%) \\
\hline fev/06 & 16 & 4894 & 0,33 \\
\hline abr/06 & 12 & 2435 & 0,49 \\
\hline jun/06 & 11 & 13268 & 0,08 \\
\hline ago/06 & 19 & 119366 & 0,02 \\
\hline out/06 & 77 & 49483 & 0,16 \\
dez/06 & 5 & 7975 & 0,06 \\
\hline Total & 140 & 197421 & 0,07 \\
\hline
\end{tabular}

A amostragem nas galhadas armadas, consequentemente nos pontos próximos ao lago Amanã e Urini, constituiu o método mais eficiente de coleta da espécie, representando $96 \%$ das capturas. O restante foi capturado por meio de arrasto nos igarapés de terra firme.

Através da relação peso-comprimento (Figura 2) e do teste $\mathrm{t}$ de Student $(\mathrm{H0}: \mathrm{b}=3)$ com nível de confiança de p de 5\% ( $a=0.05$ ), o crescimento de $H$. efasciatus pode ser considerado do tipo isométrico $(b=3,062, p=0,708)$.

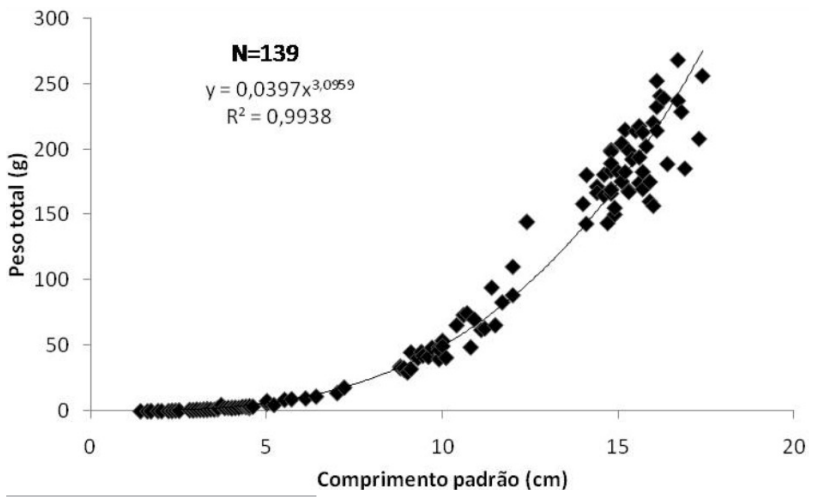

Figura 2- Relação peso x comprimento de Heros efasciatus.

Foram identificados 50 fêmeas e 42 machos, sendo a diferença entre a proporção sexual não significativa tanto para o período como um todo $(\mathrm{G}=0,70, \mathrm{df}=1, \mathrm{p}<0,404)$, quanto por mês de coleta (Tabela 2) . Outros 47 indivíduos não tiveram seu sexo determinado em função do pequeno tamanho.

Tabela 2 - Número de fêmeas e machos, proporção sexual e teste G por mês de coleta. 0 mês de fevereiro não está representado por só se ter amostrado indivíduos no qual a distinção sexual não pode ser realizada.

\begin{tabular}{lccccc}
\hline \multirow{2}{*}{ Mês } & \multicolumn{3}{c}{ Número } & \multicolumn{3}{c}{ Proporção } \\
\cline { 2 - 6 } & Fêmeas & Machos & Fêmeas & Machos & Teste G \\
\hline Abril & 4 & 3 & 0,57 & 0,43 & $\mathrm{p}=0,71$ \\
Junho & 3 & 3 & 0,50 & 0,50 & $\mathrm{p}=1,00$ \\
Agosto & 2 & 5 & 0,29 & 0,71 & $\mathrm{p}=0,25$ \\
Outubro & 38 & 29 & 0,57 & 0,43 & $\mathrm{p}=0,27$ \\
Dezembro & 3 & 2 & 0,60 & 0,40 & $\mathrm{p}=0,65$ \\
Total & 50 & 42 & 0,54 & 0,46 & $\mathrm{p}=0,40$ \\
\hline
\end{tabular}

O comprimento padrão máximo encontrado foi de 164 $\mathrm{mm}$ para as fêmeas e de $174 \mathrm{~mm}$ para os machos, enquanto os menores exemplares identificados quanto ao sexo apresentaram $36 \mathrm{~mm}$ e $31 \mathrm{~mm}$ para fêmeas e machos respectivamente. $\mathrm{O}$ comprimento médio de primeira maturaçáo $\left(\mathrm{L}_{50}\right)$ foi estimado em $97 \mathrm{~mm}$ para as fêmeas.

Para as sete gônadas femininas analisadas, a fecundidade do último lote de ovócito variou de 1704 a 3280 . Em cada ovário, foram observados ovócitos em três lotes de tamanho e em aproximadamente mesmo número (Tabela 3). Em algumas gônadas não foi possível dissociar bem os ovócitos para a contagem dos mesmos dos lotes T1 e T2

De maneira geral, indivíduos em maturação foram capturados a partir de agosto, enquanto indivíduos maduros e esgotados/desovados foram registrados em outubro e dezembro, coincidindo com os menores níveis da água (Figura 3). Entre fevereiro e junho só foram amostrados indivíduos imaturos. 
Tabela 3 - Número de ovócitos de cada lote de tamanho (T1: 0,10-0,50mm, T2: 0,50-1,00mm e T3: 1,00-1,50mm), o comprimento padrão e o peso da gônada das fêmeas analisadas.

\begin{tabular}{lcccc}
\hline $\begin{array}{l}\text { Tamanho do } \\
\text { indivíduo }(\mathrm{cm})\end{array}$ & $\begin{array}{c}\text { Peso gônada } \\
(\mathrm{g})\end{array}$ & $\begin{array}{c}\mathrm{n}^{\circ} \text { ovócitos } \\
\mathrm{T} 1\end{array}$ & $\begin{array}{c}\mathrm{n}^{\circ} \text { ovócitos } \\
\text { T2 }\end{array}$ & $\begin{array}{c}\mathrm{n}^{\circ} \text { ovócitos } \\
\text { T3 }\end{array}$ \\
\hline 14,4 & 3,58 & 2600 & 1400 & 2660 \\
14,6 & 1,9 & - & - & 2494 \\
14,9 & 3,134 & 2497 & 3349 & 2808 \\
14,9 & 3,134 & - & - & 1704 \\
15,2 & 2,97 & - & - & 2482 \\
15,3 & 2,122 & 2710 & 1240 & 2090 \\
15,9 & 2,25 & 6820 & 4520 & 3280 \\
\hline
\end{tabular}

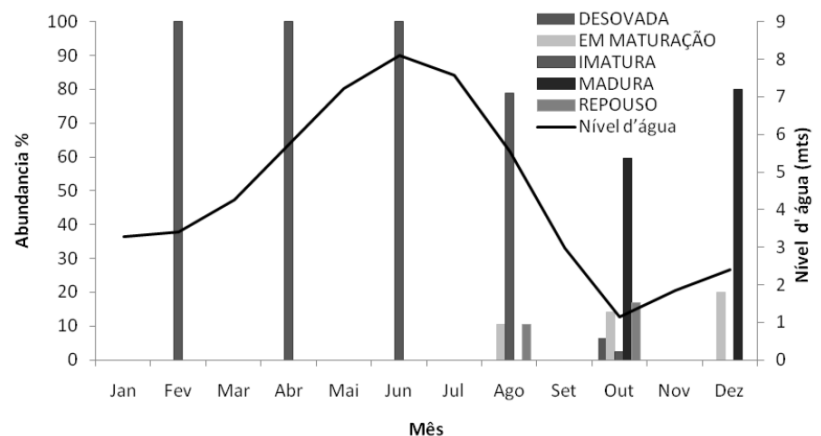

Figura 3 - Nível d'água médio entre dezembro de 2001 e outubro de 2005, frequencia dos estádios de maturação para sexos agrupados ao longo de um ano.

A relação gonadossomática e o índice gonadal apresentaram valores mais elevados em outubro e dezembro (Figura 4 e 5). Já o fator de condição alométrico variou significativamente entre as coletas $(K=4,70 ; p<0,001)$, apresentando menores valores em abril e junho (Figura 6).

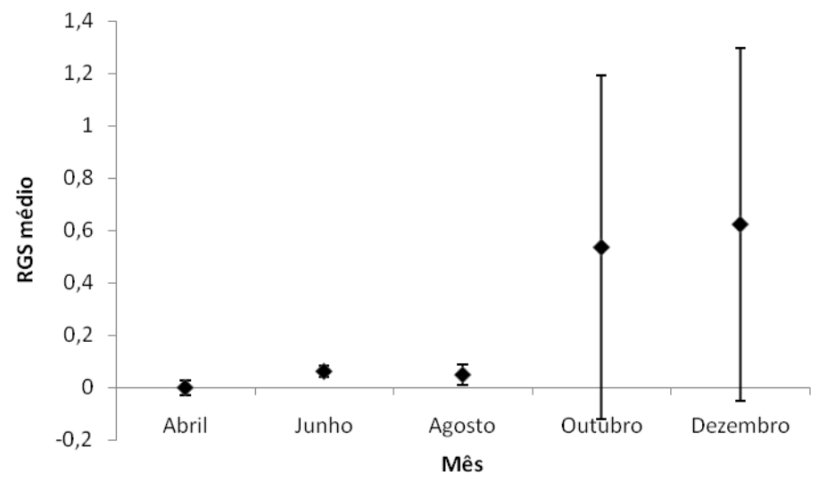

Figura 4 - Relação gonadossomática (RGS) média para cada período amostrado, para sexos agrupados.

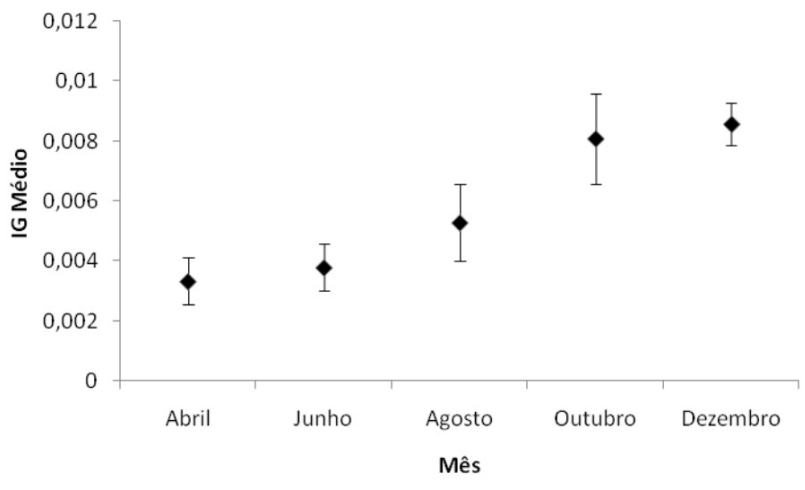

Figura 5 - Índice gonadal (IG) médio para cada período amostrado, para sexos agrupados.

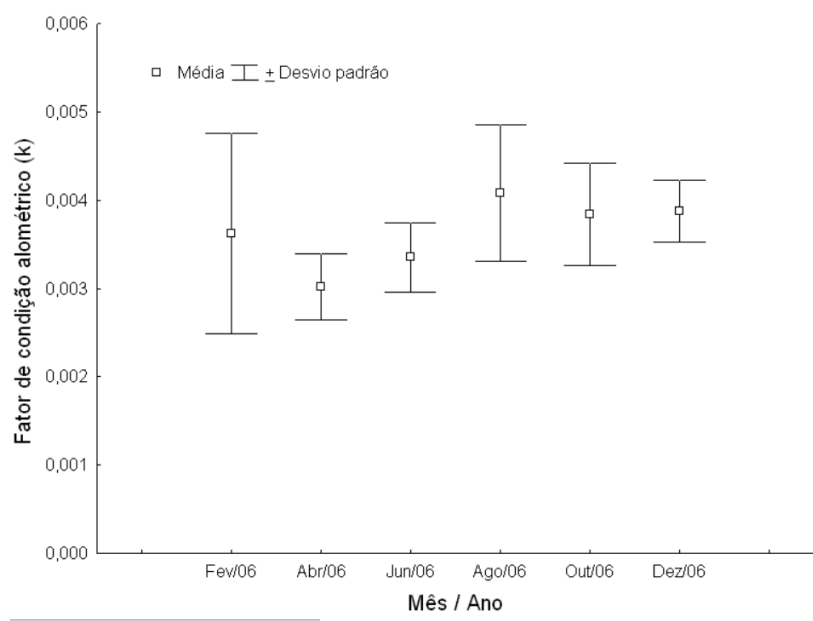

Figura 6 - Média e desvio padrão do fator de condição alométrico (K) para cada período amostrado, para sexos agrupados.

$\mathrm{Na}$ regiáo de estudo, indivíduos de pequeno porte (menor que $8 \mathrm{~cm}$ ) foram capturados entre fevereiro e junho. Em agosto já foram obtidos alguns individuos maiores que $8 \mathrm{~cm}$, porém nos meses de outubro e dezembro foi quando se coletou com maior frequencia os representantes das maiores classes de tamanho (Figura 7).

\section{DISCUSSÃO}

O aumento da relação gonadossomática e do índice gonadal em outubro e dezembro reflete um aumento do peso das gônadas, sendo possível inferir que o período reprodutivo de H. efasciatus seja durante esta época. Além disso, indivíduos maduros foram amostrados em outubro, pico da seca, e em dezembro, quando entáo já se nota uma elevação do nível d'água. Deste modo, provavelmente os ovos eclodirão na época da enchente, assegurando aos filhotes maior disponibilidade de oxigênio, minimizando os riscos de predação da prole devido ao aumento de abrigos e maximizando a quantidade de alimento (Vazzoller 1996). Vários estudos sobre a biologia 

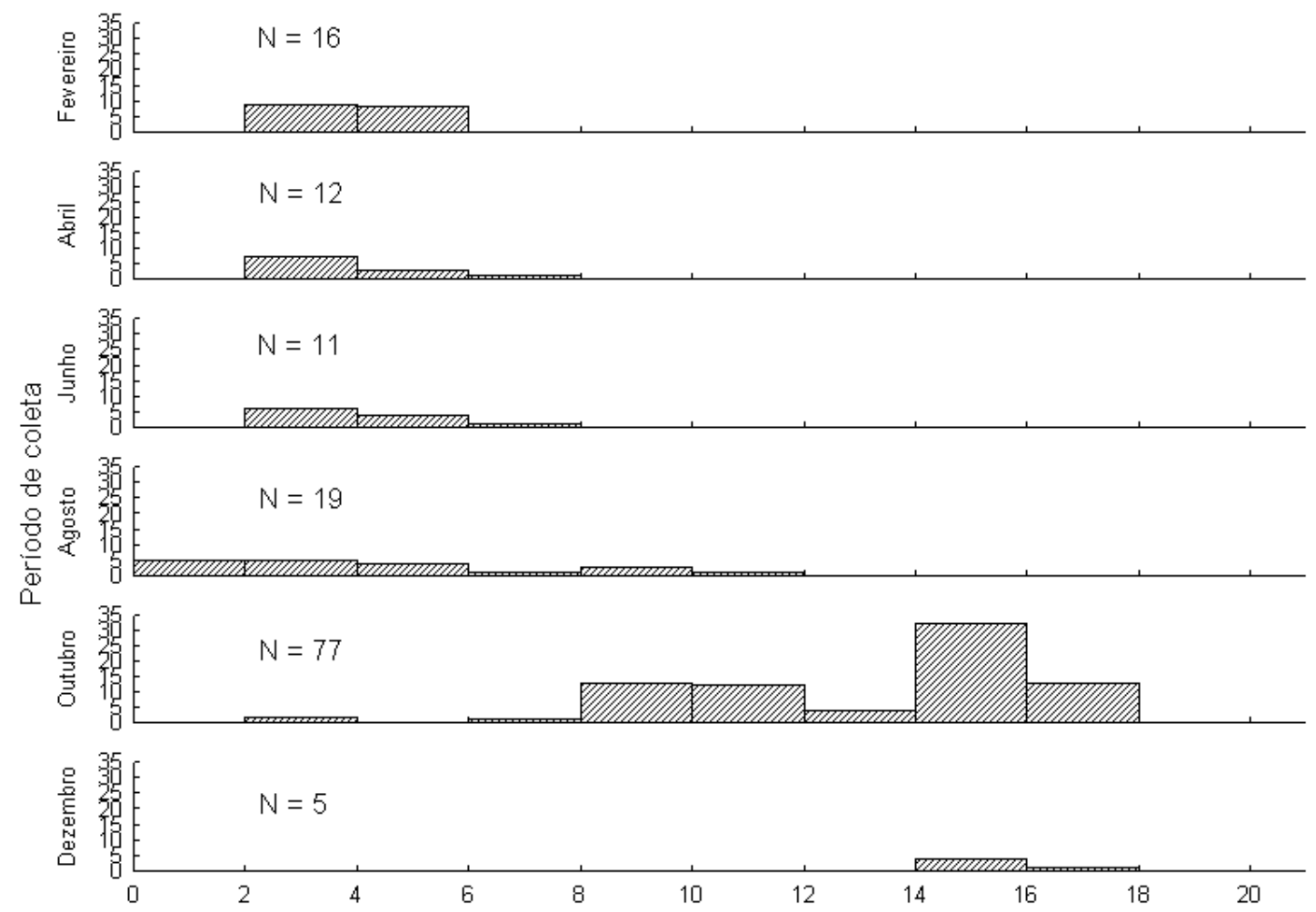

Classes de tamanho (cm)

Figura 7 - Histogramas de frequência de comprimento $(\mathrm{cm})$ dos indivíduos por período de coleta.

reprodutiva de peixes da Amazônia têm apontado o período de enchente como sendo a fase do ciclo hidrológico onde a maioria das espécies se reproduz (Santos e Ferreira 1999; Isaac et al. 2000; Santos 2006).

O fator de condição $(\mathrm{K})$ reflete as condiçôes alimentares e reprodutivas recentes (Vazzoller 1996), uma vez que grande parte da energia acumulada pelo indivíduo deve ter sido canalizada para o desenvolvimento das gônadas e/ou para migração ou outros comportamentos reprodutivos. Assim, o período delimitado pelos valores mais elevados de $\mathrm{K}$, (agosto a dezembro) deve coicidir com o período reprodutivo da espécie. A diminuição dos valores de $\mathrm{K}$ a partir de dezembro pode estar relacionada à diminuiçấo do peso dos indivíduos após a reprodução (p.e. desova). Santos (2006) estudando Hoplosternum littorale mostrou que o fator de condiçâo de tal espécie apresentou valores significativamente maiores no estádio maduro e nos períodos de enchente e cheia. Moreira (2004), ao analisar a condição de Psectrogaster rutiloides em área de várzea, também registrou maiores valores do fator de condiçáo nos períodos reprodutivos, sendo igualmente utilizado pela autora para confirmar o período reprodutivo da espécie.
Como a diferença na proporção sexual encontrada náo foi significamente diferente, ela está dentro da razão teórica esperada (proporção sexual de 1:1).

Nos ciclídeos a fecundidade é muito variável. Em representantes de grande porte, como o tucunaré (Cichla monoculus), a fecundidade por cada lote de ovócitos é de cerca de 3100 (Chellapa et al. 2003), enquanto de pequeno porte, como o acará-disco (Symphysodon discus), apresenta fecundidade média de 536 ovócitos por lote (Câmara 2004). Assim sendo, a fecundidade média calculada em 2502 ovócitos indica que a espécie possui baixa fecundidade. Como foram observados ovócitos em três estágios de tamanho e em aproximadamente mesmo número em cada fêmea analisada, sugere-se que o mecanismo de desenvolvimento ovocitário seja sincrônico em mais de dois grupos, tipo de desenvolvimento que caracteriza uma desova múltipla ou parcelada (Vazzoller 1996). A provável desova parcelada de $H$. efasciatus acompanha o padrão encontrado na literatura para a maioria das espécies de ciclídeos (Keenleyside 1991).

As informaçôes levantadas sobre a espécie contribuirão na adoção de uma série de medidas de manejo que tenham como objetivo a sustentabilidade de sua exploração pesqueira. 
Entre estas informaçôes, podem ser destacadas a determinaçáo de tamanhos mínimos de captura, superiores aos tamanhos médios de maturação encontrados ( $97 \mathrm{~mm}$ para as fêmeas) como forma de tentar garantir que cada indivíduo reproduza pelo menos uma vez antes de sua captura. Outra medida que poderia ser adotada é o estabelecimento de períodos de defeso, durante a época de reprodução da espécie (outubro a janeiro), impedindo que capturem indivíduos ovados ou pais em fase de cuidado da prole, característica esta encontrada em muitos ciclídeos, sendo que a prole pode não se desenvolver sem o cuidado parental (Goldstein 1973).

A pequena abundância de indivíduos na regiáo amostrada indica que, para esta localidade, deve-se capturar anualmente um pequeno número de indivíduos dessa espécie, garantindo a continuidade do estoque. A baixa fecundidade média aponta para a necessidade de acompanhamento constante de suas populaçóes uma vez implementado o manejo, já que uma eventual recuperação de estoques se daria de maneira mais lenta.

Apesar de diversos autores como West (1990) e Dias et al. (1998), apontarem diversos problemas na classificação do desenvolvimento gonadal macroscopicamente, uma vez que a mesma é mais sujeita a erros, e defenderem o uso da microscopia para uma maior acuidade, o presente trabalho, por fazer parte de um projeto de levantamento da ictiofauna nos igarapés da RDS Amaná, visou aproveitar o material já coletado e fixado, justificando as análises realizadas, evitando que fossem sacrificados mais exemplares. Além disso, para a finalidade de obter dados para o manejo, apenas a análise macroscópica foi suficiente. Dias et al. (1998), sugerem alguns procedimentos quando a análise microscópica não pode ser realizada, como um levantamento prévio de informaçôes disponíveis sobre a biologia das espécies ou de espécies congenéricas, utilização de escalas de classificação dos ovários simplificada e análise simultânea das variaçôes de outros indicadores de maturaçáo, que foram adotados.

Por se tratar de uma espécie com potencial para a ornamentação pouco estudada e já comercializada e valorizada no mercado aquarista, estudos como este sáo importantes ao fornecer dados biológicos básicos que poderão ser úteis para o manejo sustentável da espécie.

\section{AGRADECIMENTOS}

A toda equipe do Instituto de Desenvolvimento Sustentável Mamirauá pelo apoio logístico e financeiro, em especial aos participantes do Projeto "Peixes ornamentais" que contribuíram de diversas maneiras para a realizaçáo deste estudo. Ao CNPq pela bolsa concedida ao segundo autor.

\section{BIBLIOGRAFIA CITADA}

Ayres, J. 1993. Mamirauá flooded forests. MCT-CNPq-PTU, Sociedade Civil Mamirauá, Belém, PA. 123 pp (in Portuguese).

Batista, V.S.; Petrere Júnior, M. 2003. Characterization of the commercial fish production landed at Manaus, Amazonas State, Brazil. Acta Amazonica, 33(1): 53-66 (in Portuguese, with abstract in English).

Câmara, M.R. 2004. Reproductive biology of an ornamental neotropical cichlid fish, Symphysodum discus Heckel, 1980 (Osteichthyes: Perciformes: Cichlidae). Tese de Doutorado, Universidade Federal de São Carlos, São Carlos, São Paulo. 147 pp (in Portuguese, with abstract in English)

Cáuper, G.C.B. 2006. Biodiversidade Amazônica-Flora Amazônica. Amazonian Biodiversity - Amazonian Flora. Centro Cultural dos Povos da Amazônia, Manaus, Amazonas. 110 pp (in Portuguese).

Chellapa,S.; Câmara,M.R.; Chellappa,N.T; Beveridge,M.C.M.; Huntingford, F.A. 2003. Reproductive ecology of a neotropical cichlid fish, Cichla monoculus (Osteichthyes:Cichlidae). Brazilian Journal of Biology, 63:17-26.

Dias, J.F.; Peres-Rio E.; Chaves, P.T.C.; Rossi-Wongtschowski, C.L.D.B. 1998. Macroscopical Analysis of Ovaries of Teleosts: Problems of classification and Recommended Procedures. Revista Brasileira de Biologia, 58 (1):55-69 (in Portuguese, with abstract in English).

Fleck, L.C. 2004. Wildlife use and conservation in the Boa Esperança community, Amanã Reserve, Amazon. Dissertação de Mestrado, Durrell Institute of Conservation and Ecology/University of Kent, UK. 102 p.

Froese, R.; Pauly, D. (Eds.). FishBase. (www.fishbase.org). Acesso em: 15 nov. 2009.

Goldstein, R.J. 1973. Cichlids of the World. T.H.F. Publications, Neptune City, USA, 382 p.

Isaac, V.J.; Rocha, V.L.C.; Mota, S.Q.C. 2000. Reproductive cycle of some commercial fish species from lower Amazonas river, p. 31-61. In: Isaac, V. J.; Rocha, V. L. C.; Mota, S. Q. C. (Eds). Fisheries Resources from Medium Amazonas river-Biology and Fishing Stastistics. Vol. 22. Instituto Brasileiro do Meio Ambiente e dos Recursos Naturais Renováveis, Série Estudo Pesca, Coleção Meio Ambiente. Brasília, DF. (in Portuguese).

Keenleyside, M.H.A. 1991. Cichlid Fishes-Behaviour Ecology and Evolution. Chapman and Hall, London, UK, 377 p.

Le Cren, E.D. 1951. The lenght-weight relationship and seasonal cycle in gonad and conditions in the perch Perca fluviatilis. Journal Animal Ecology, 20 (2): 201-219.

Lowe- McConnell, R.H. 1999. Ecological studies of tropical fish communities. EDUSP, São Paulo, SP. 534 pp (in Portuguese).

Moreira, S.S. 2004. Relations between the hydrological cycle, feeding activity and reproductive tactics of four fish species from Cataläo, Central Amazonian. Dissertação de Mestrado. Instituto Nacional de Pesquisas da Amazônia/ Universidade Federal do Amazonas. Manaus, 108 pp (in Portuguese). 
Nogueira, V. de P.Q. 1997. Criation Proposal: Reserve of Sustantable Management Amanã. [s.l.]: IPAAM, Tefé, Amazonas 9 pp (in Portuguese).

Santos, R.N. 2006. Influence of the hydrological cycle, gonadal maturation and trophic category in the energy of fish from a flooded Forest at Central Amazonian. Dissertação de Mestrado. Instituto Nacional de Pesquisas da Amazônia/Universidade Federal do Amazonas, Manaus, 74 pp (in Portuguese).

Santos, G.M.; Ferreira, E. 1999. Amazonian Fish, p. 220-250 In: Lowe-MacConnell, R. H (Ed). Ecological studies of tropical fish communities. EDUSP, São Paulo, São Paulo (in Portuguese)

Sokal, R.R.; Rohlf, F.J. 1987. Introduction to biostatistics. Freeman Publication, New York, USA, 363 pp.
Vazzoler, A.E.A.M. 1996. Reproduction Biology of teleosts fish; Theory and Practical. Eduem, Maringá, Paraná, 169 pp.

West, G. 1990. Methods of assessing ovarian development in Fishes: a review. Australian Journal of Marine and Freshwater Research, 41:199-222.

Zar, J.H. 1999. Biostatistical analysis. $4^{\mathrm{a}} \mathrm{ed}$. New Jersey, PrenticeHall, 663 pp.

Recebido em 27/04/2009

Aceito em 10/12/2009 